\title{
Quantifying the potential for mineral carbonation of processed kimberlite with the Rietveld-PONKCS method
}

\author{
BAOLIN WANG ${ }^{1}$, NINA ZEYEN ${ }^{2}$, SIOBHAN WILSON ${ }^{1}$, \\ REBECCA FUNK ${ }^{1}$ AND CONNOR TURVEY ${ }^{3}$ \\ ${ }^{1}$ University of Alberta \\ ${ }^{2}$ University of Alberta - EAS \\ ${ }^{3}$ University of British Columbia \\ Presenting Author: baolin1@ualberta.ca
}

Quantitative phase analysis (QPA) using the Rietveld method and X-ray diffraction (XRD) patterns is useful for predicting the reactivity of a rock to $\mathrm{CO}_{2}$ and for quantifying mineral carbonation [1]. Kimberlites can be reactive to $\mathrm{CO}_{2}$, but they are rich in structurally disordered clay minerals. In this study, the method of Partial Or No Known Crystal Structure (PONKCS) [2] was used to model the peak profiles of smectite(s) and lizardite to account for turbostratic stacking disorder in synthetic and natural samples of processed kimberlite.

Here we used weighed mixtures to develop an accurate approach to QPA for kimberlite. Lizardite and montmorillonite PONKCS models were made using XRD patterns collected with a Rigaku Ultima IV, a Bruker D8 Advance and a Bruker D8 Advance Eco. PONKCS models were prepared from 50:50 wt\% mixtures of lizardite and Ca-exchanged montmorillonite (SWy2) with NIST or Baikalox $\alpha-\mathrm{Al}_{2} \mathrm{O}_{3}$. Five synthetic samples of processed kimberlite of typical and known composition were then used to test the results of these PONKCS models for all three XRDs. We also tested the sensitivity of the PONKCS method to changes in instrument geometry. PONKCS models calibrated for one instrument were used in refinements using XRD data collected on a different instrument, or on the same instrument with modified geometry. Our results show that even small changes in instrument parameters can lead to inconsistent and inaccurate QPA results using PONKCS $(9.8-32.5 \%$ total bias [3] for our weighed mixtures). However, the results can be highly accurate (4.2-14.1\% total bias) using correctly calibrated, instrument specific PONKCS models. Finally, we use this approach to quantify the mineralogy of kimberlites from laboratory-based acid leaching experiments and from a threetonne field trial as part of De Beers' Project CarbonVault.

[1] Wilson, S.A. et al. (2006). Am Mineral 91: 1331-1341.

[2] Scarlett, N.V.Y., I.C. Madsen (2006). Powder Diffraction 21: $278-284$.

[3] Omotoso, O. et al. (2006). Clays Clay Mineral, 54:748760 . 